\title{
Detailed chemical composition of the open cluster IC 4651: The iron peak, $\alpha$ elements, and $\mathrm{Li}^{\star}$
}

\author{
L. Pasquini ${ }^{1}$, S. Randich ${ }^{2}$, M. Zoccali ${ }^{1}$, V. Hill ${ }^{3}$, C. Charbonnel ${ }^{4,5}$, and B. Nordström ${ }^{6,7}$ \\ ${ }^{1}$ European Southern Observatory, 85748 Garching bei München, Germany \\ e-mail: lpasquin@eso.org \\ 2 INAF - Osservatorio di Arcetri, Largo Enrico Fermi 5, 50125 Firenze, Italy \\ 3 GEPI (URA8111), Observatoire de Paris-Meudon, 92195 Meudon, France \\ 4 Observatoire de Genève, 1290 Sauverny, Switzerland \\ 5 LATT, OMP, CNRS UMR 5572, 14, av. E. Belin, 31400 Toulouse, France \\ ${ }^{6}$ Niels Bohr Institute for Astronomy, Physics \& Geophysics, Juliane Maries Vej 30, 2100 Copenhagen, Denmark \\ 7 Lund Observatory, Box 43, 22100 Lund, Sweden
}

Received 10 February 2004 / Accepted 28 May 2004

\begin{abstract}
We present a detailed chemical analysis of 22 stars along the colour-magnitude sequence of the intermediate-age (1.7 Gyr) open cluster IC 4651, based on high-resolution, high $\mathrm{S} / \mathrm{N}$ ratio spectra from UVES/VLT. IC 4651 thus becomes one of the few open clusters for which a detailed composition analysis exists for stars spanning $3.5 \mathrm{mag}$, from solar-type mainsequence stars to giants above the RGB clump. In a strict comparison with the Sun, we find for the cluster a well-defined Fe abundance of $[\mathrm{Fe} / \mathrm{H}]=0.10 \pm 0.03$ (internal errors), with a reddening $E(b-y)=0.091$. We also derive abundances for the $\alpha$ elements $\mathrm{Mg}, \mathrm{Si}$, Ti, and $\mathrm{Ca}$ and find a moderate enhancement of the three former elements, in excellent agreement with the results for field stars of similar Fe abundance. Among the Fe group elements, $\mathrm{Cr}$ and $\mathrm{Ni}$ are slightly overabundant $([\mathrm{X} / \mathrm{Fe}] \sim$ 0.05). As also observed in M 67, the $\mathrm{Na}$ abundance among the giants is more than 0.2 dex higher than in the dwarfs. We interpret this effect as real, and due to dredge-up of ${ }^{23} \mathrm{Na}$ in the giants.

Four turnoff stars, all fairly rapid rotators $\left(v \sin i \geq 25 \mathrm{~km} \mathrm{~s}^{-1}\right)$, appear to have higher $[\mathrm{Fe} / \mathrm{H}]$ than the other stars; our tests show that, while a spurious enhancement of 0.1 dex can be produced by the effect of high rotation on our spectral analysis, this cannot explain the whole difference. These stars appear overabundant because we tend to overestimate their effective temperatures by forcing excitation equilibrium.

Li abundances have been computed for all the stars and show a well-defined pattern: the Solar-type stars have an almost constant $\mathrm{Li}$ abundance, just below that of the Hyades, and the Li-dip is pronounced and well determined. Turnoff stars just above the dip have a "cosmic" Li abundance, but within a very small range of magnitudes $(0.25 \mathrm{mag})$ higher on the turnoff, the Li level drops by more than a factor 10. This cannot be due just to dilution; rather some extra mixing is required. Among the giants, two probable clump stars show detectable Li, while all the other (likely) RGB stars do not - as is also observed in the similar cluster NGC 3680. None of these patterns can be explained by classical stellar evolution models. Again, some extra mixing is required. We show that rotating stellar models including the most recent developments for meridional circulation and turbulence by shear instabilities explain very well the behaviour of the lithium abundance along the colour-magnitude diagram of IC 4651, including subgiant and giant phases. The possibility remains open that the giants exhibiting the highest lithium abundances are actual RGB bump stars which have just been through the so-called "lithium flash".
\end{abstract}

Key words. stars: abundances - Galaxy: open clusters and associations: individual: IC 4651 - stars: evolution - stars: rotation

\section{Introduction}

Open clusters are key tracers of the chemical evolution of the Galaxy and, at the same time, benchmarks for stellar evolution models. Despite the many advances in the detailed modelling of stellar evolution over the last few years (see e.g. Girardi et al. 2000), very few open clusters have been studied in a comprehensive manner. Membership studies have become available

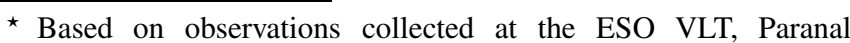
Observatory, Chile. for several clusters, but the number of detailed chemical analyses remains very small (e.g., Gratton 2000, and references therein).

The determination of heavy-element abundances and abundance ratios and their variation with time and Galactocentric distance is crucial for our understanding of the formation and chemical evolution of the Galactic disk (e.g., Edvardsson et al. 1993; Tosi 2000). Abundances in clusters also provide a fundamental tool to constrain the relative importance of type I and type II supernovae in the enrichment of the interstellar medium 
and thus to gain insight in the star formation history in the disk. On the other hand, the abundance evolution of the fragile elements $\mathrm{Li}$ and $\mathrm{Be}$ along the colour magnitude diagram (CMD) of a cluster represents a precious testbench for stellar evolutionary theories, as the initial stellar mass is the only variable parameter within a cluster (see e.g., Pasquini 2000; Deliyannis et al. 2000).

For these reasons, we decided to carry out a detailed abundance analysis of the rich, well-studied, intermediate age cluster IC 4651, based on high-quality spectra for 23 members obtained with the VLT/UVES. The Strömgren photometry by Meibom (2000) and radial-velocity survey by Meibom et al. (2002) allowed us to select target stars known to be members, single, and with known rotational velocity. It also provided the basic properties of the cluster (distance, reddening, overall metallicity, and age).

In the present paper, we briefly summarize the properties of the cluster and describe the observations, data reduction and analysis. We then discuss the metal and $\alpha$ element abundances, while the last section before the conclusions is devoted to a detailed analysis of the evolution of lithium. The abundances of $\mathrm{O}$ and $\mathrm{Be}$ and the $\mathrm{C}^{12} / \mathrm{C}^{13}$ isotopic ratio will be the subject of a future paper.

\section{Basic properties of IC 4651}

The early studies of IC 4651 by Eggen (1971), Lindoff (1972), and Anthony-Twarog et al. (1987, 1988, 2000) were superseded by the extensive uvby photometry of 17640 stars by Meibom (2000), supplemented by the radial-velocity survey of 104 stars by Meibom et al. (2002). Notably, the wide field observed by Meibom (2000) roughly doubled the area and stellar content of the cluster relative to earlier studies; because the radial-velocity survey was initiated about a decade earlier, it is complete for the inner cluster only (to $V \sim 14.5$ ).

Meibom et al. (2002) found IC 4651 to have a reddening of $E(B-V)=0.10$, a distance of $1 \mathrm{kpc}\left((m-M)_{0}=10.03 \pm\right.$ $0.1)$, a metal abundance of $[\mathrm{Fe} / \mathrm{H}]=+0.1 \mathrm{dex}$, and an age of $1.7 \pm 0.15 \mathrm{Gyr}$; the mass of the cluster stars at the turnoff (TO) is $1.8 M_{\odot}$. The present stellar population of IC 4651 was estimated to be $\sim 650$ stars with a total mass of $\sim 630 M_{\odot}$. IC 4651 was, however, also found to show moderate mass segregation. A comparison of the present and plausible initial mass functions indicated that it initially contained at least $\sim 8300$ stars with a total mass of $\sim 5300 M_{\odot}$. Of this mass, $\sim 35 \%$ has been lost during the evolution of the initially most massive stars, while the remaining $\sim 53 \%$ ( $\sim 93 \%$ of the original low-mass stars) have evaporated from (the central part of) the cluster.

From its space motion, Meibom et al. (2002) also computed the Galactic orbit of IC 4651. They found a fairly eccentric orbit $(e=0.19)$, with a mean galactocentric orbital radius of $8.6 \mathrm{kpc}$ and peri- and apogalactic distances 7.0 and $10.2 \mathrm{kpc}$, respectively. IC 4651 is currently $7.1 \mathrm{kpc}$ from the Galactic centre and 140 pc below the Galactic plane; its maximum distance from the plane is $190 \mathrm{pc}$.

The conclusion of Meibom et al. (2002) that IC 4651 is metal rich $([\mathrm{Fe} / \mathrm{H}]=+0.1 \mathrm{dex})$ was based on the same type of $u v b y \beta$ photometry as used by Nordström et al. (1997)

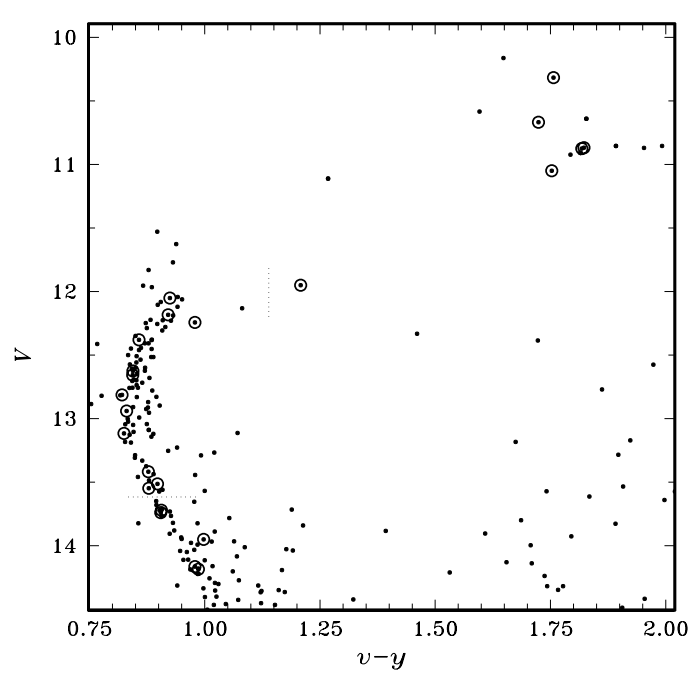

Fig. 1. Colour-magnitude diagram of IC 4651 from Meibom et al. (2002); our programme stars are indicated by circles.

to derive a similarly high metallicity for the roughly coeval cluster NGC 3680. However, for the latter cluster, Pasquini et al. (2001) found $[\mathrm{Fe} / \mathrm{H}]=-0.17$ from high-resolution spectroscopy, a result that appears to be corroborated by the recent photometry of Anthony-Twarog \& Twarog (2004). Thus, one goal of the present study is to check the iron abundance of IC 4651 by careful high-resolution spectroscopy.

\section{Sample selection and observations}

20 programme stars were selected for observation from the unpublished thesis by Meibom, pending the publication of the detailed results by Meibom (2000) and Meibom et al. (2002). Stars were chosen to be single and among the slower rotators in each magnitude bin, since an accurate analysis of very broadlined spectra is not possible, in particular in the Be region. The stars were also chosen to sample a large section of the CMD; based on our previous experience with the twin cluster NGC 3680 (Pasquini et al. 2001), it was clear that the region around the so called Li-dip (Boesgaard \& Tripicco 1986) at the turnoff (TO) would be particularly interesting; thus we took care to sample this region more densely than was possible in the more sparsely populated NGC 3680 (Nordström et al. 1997). To these 20 stars we added the three stars previously analyzed by Randich et al. (2002), which were also observed with UVES in dichroic mode.

Our sample (including the stars from Randich et al. 2002) is listed in Table 1. Numbers less than 100 and with prefix " $E$ " are from Eggen (1971); higher numbers with prefix "T" are from Anthony-Twarog et al. (1988). Strömgren photometry and rotational velocities are from Meibom (2000) and Meibom et al. (2002), while the $B-V$ colors are from Anthony-Twarog et al. (1988); the $v$ magnitude for E98 by Meibom (2000) has been corrected for an apparent typographical error. The CMD of IC 4651 is shown in Fig. 1, with our sample stars circled for identification.

The observations were carried out at the ESO VLT Kueyen telescope and its UVES spectrograph (Dekker et al. 2000). We 
Table 1. Our sample stars in the giant, turnoff, and lower mainsequence regions. The Strömgren photometry is from Meibom (2000), $B-V$ from Twarog et al. (1988), and $v \sin i\left(\mathrm{~km} \mathrm{~s}^{-1}\right)$ from Meibom et al. (2002).

\begin{tabular}{lcccccr}
\hline \hline Name & $y$ & $b-y$ & $m_{1}$ & $c_{1}$ & $B-V$ & $v \sin i$ \\
\hline E12 & 10.324 & 0.661 & 0.465 & 0.314 & 1.126 & 1.1 \\
E8 & 10.667 & 0.666 & 0.403 & 0.299 & 1.100 & 0.1 \\
E60 & 10.870 & 0.673 & 0.491 & 0.312 & 1.141 & 1.9 \\
E98 & 10.880 & 0.694 & 0.496 & 0.331 & 1.187 & 1.0 \\
T812 & 11.059 & 0.648 & 0.413 & 0.344 & 1.140 & 0.5 \\
E95 & 11.953 & 0.514 & 0.185 & 0.386 & 0.805 & 12.0 \\
\hline E3 & 12.051 & 0.374 & 0.169 & 0.527 & 0.612 & 29.9 \\
E56 & 12.184 & 0.378 & 0.159 & 0.499 & 0.599 & 27.8 \\
E19 & 12.240 & 0.412 & 0.157 & 0.484 & 0.651 & 32.1 \\
E99 & 12.377 & 0.347 & 0.171 & 0.531 & 0.546 & 28.1 \\
E17 & 12.625 & 0.337 & 0.158 & 0.543 & 0.539 & 10.0 \\
E25 & 12.654 & 0.365 & 0.118 & 0.552 & 0.537 & 21.3 \\
E5 & 12.817 & 0.329 & 0.160 & 0.532 & 0.507 & 23.9 \\
T1228 & 12.941 & 0.344 & 0.130 & 0.540 & 0.536 & 5.7 \\
E14 & 13.119 & 0.335 & 0.154 & 0.507 & 0.516 & 34.1 \\
E34 & 13.418 & 0.358 & 0.156 & 0.459 & 0.566 & 25.2 \\
E15 & 13.515 & 0.374 & 0.146 & 0.447 & 0.563 & 10.0 \\
E79 & 13.549 & 0.360 & 0.154 & 0.444 & 0.514 & 21.8 \\
\hline E64 & 13.722 & 0.370 & 0.160 & 0.430 & 0.594 & 9.2 \\
E86 & 13.739 & 0.383 & 0.143 & 0.431 & 0.626 & 14.0 \\
T2105 & 13.948 & 0.429 & 0.145 & 0.374 & 0.618 & 11.3 \\
E7 & 14.165 & 0.402 & 0.179 & 0.368 & 0.643 & 2.1 \\
E45 & 14.184 & 0.430 & 0.127 & 0.399 & 0.654 & 4.2 \\
\hline & & & & & &
\end{tabular}

used UVES in dichroic mode, which allowed us to simultaneously observe the near-UV/blue and red spectral ranges. In order to obtain a good signal in the region of the UV Be lines $(313 \mathrm{~nm})$, much longer exposure times are required than at longer wavelengths. We thus chose to obtain several red exposures, using both CD\#3 centered at $580 \mathrm{~nm}$ and CD\#4 centered at $860 \mathrm{~nm}$, simultaneously with the UV ones; in this way we were able to observe the whole range $480-1000 \mathrm{~nm}$ in all the stars, with a gap in spectral coverage from 390 to $480 \mathrm{~nm}$ and very small gaps around 580 and $860 \mathrm{~nm}$.

The three stars previously analyzed by Randich et al. (2002), which were also observed with UVES in dichroic mode, had several red exposures each in the setup centered around $580 \mathrm{~nm}$. Each of these exposures was used to compute independent abundances, as if they pertained to different objects. This gives a good estimate of the internal random errors.

The observations were carried out in service mode between May and June 2001. Whereas in the UV a resolving power $R \sim 45000$ was used ( 1 arcsec slit), we opted for a very high resolution $(R=100000)$ in the red, using a 0.4 arcsec wide slit. The typical $S / N$ ratio of our spectra is $70-120$ per pixel. The data of Randich et al. were characterized by a similar $S / N$ ratio, but a lower resolution $(R \sim 45000)$.

Both raw data and spectra reduced using the ESOUVES pipeline (Ballester et al. 2000) were sent to us; after independently re-reducing several sample spectra we concluded that a complete, independent re-reduction was not warranted.

\section{Data analysis: Stellar parameters and determination of abundances}

\subsection{Stellar parameters and iron abundances}

Initial effective temperatures $\left(T_{\text {eff }}\right)$ were derived following the Alonso et al. $(1996,1999)$ scales, based on $(b-y)$ colours and assuming a reddening of $E(b-y)=0.072$ (Meibom et al. 2002). Using a TO mass of $1.8 M_{\odot}$ and a distance modulus of $(m-M)_{0}=10.03$ (Meibom et al. 2002), we then computed surface gravities $\left(\log g\right.$ ) from mass, luminosity and $T_{\text {eff. For each }}$ star, $T_{\text {eff }}$ and the microturbulence velocity $\xi$ have then been varied so as to eliminate any dependence of the $\mathrm{Fe} \mathrm{I}$ abundance on the line excitation potential and equivalent width. Surface gravities were not varied by more than 0.3 dex. Due to the good quality of the spectra and the many lines present in our large spectral range, we could avoid using lines in the flat portion of the curve of growth, i.e., equivalent widths larger than $100 \mathrm{~m} \AA$. As mentioned, we did not attempt to vary $\log g$ in order to have $\log n\left(\mathrm{Fe}_{\mathrm{I}}\right)=\log n(\mathrm{Fe} \mathrm{II})$, but just tried to obtain an ionization balance as good as possible while keeping a gravity compatible with the position of the star in the CMD.

Our analysis was carried out differentially with respect to the Sun starting from measured equivalent widths, hence most of the systematic effects should cancel out. We shall see, however, that for some elements this may not be completely true and some differences may be present between dwarfs and giants.

Equivalent widths were measured by using an automatic line width programme: DAOSPEC (Stetson et al. 2004) and typically $80-100 \mathrm{Fe}$ I lines were used. The abundance calculations were performed with an updated and improved version of the original code described in Spite (1967). Edvardsson et al. (1993) model atmospheres were used for the dwarfs, while Gustafsson et al. (1975) models were employed for the giants. For the subgiant E95 we derived the Fe abundance using both models and found excellent agreement (within $0.01 \mathrm{dex}$ in $\mathrm{Fe} / \mathrm{H}$ ); thus, we do not expect any major spurious effect by using the two sets of model atmospheres in the two different evolutionary regimes. Finally, for most elements we used the line list of Pancino et al. (2002); atomic parameters were mainly retrieved from the National Institute of Science and Technology Atomic Spectra Database. For Ca and Al, the data were taken instead from the VALD database (Kupka et al. 1999).

\subsection{Iron abundances}

In Table 2 we list the initial photometric $T_{\text {eff }}$, the final (spectroscopic) adopted $T_{\text {eff }}$, microturbulence $\xi$, and $\log g$ values, together with the derived $\mathrm{Fe}$ I and Fe II abundances. The first line of the table reports the solar abundances that we have determined by analyzing the UVES solar spectrum (www.eso.org/instruments/UVES) in the same fashion as 
Table 2. Spectroscopic and photometric $T_{\text {eff }}$ and $[\mathrm{Fe} / \mathrm{H}]$ for the Sun and the stars in IC 4651. For the Sun, we give $\log \epsilon(\mathrm{Fe})$, while all cluster star abundances are relative to this value $([\mathrm{Fe} / \mathrm{H}])$. Values in parenthesis were omitted when computing mean abundances.

\begin{tabular}{lcccccr}
\hline \hline Star & $\begin{array}{c}T_{\text {phot }} \\
(\mathrm{K})\end{array}$ & $\begin{array}{c}T_{\text {spec }} \\
(\mathrm{K})\end{array}$ & $\log g$ & $\begin{array}{c}\xi \\
\left(\mathrm{km} \mathrm{s}^{-1)}\right.\end{array}$ & {$[\mathrm{Fe} \mathrm{I}]$} & {$[\mathrm{Fe}$ I $]$} \\
\hline Sun & 5800 & 5750 & 4.4 & 0.9 & 7.57 & 7.59 \\
\hline E12 & 4940 & 5000 & 2.7 & 1.5 & 0.09 & -0.13 \\
E8 & 4870 & 4900 & 2.7 & 1.3 & 0.09 & -0.13 \\
E60 & 4850 & 4900 & 2.9 & 1.4 & 0.07 & 0.09 \\
E98 & 4870 & 4900 & 3.0 & 1.4 & 0.15 & 0.05 \\
E812 & 4830 & 5000 & 3.0 & 1.6 & 0.11 & -0.05 \\
E95 & 5560 & 5800 & 3.5 & 1.7 & 0.07 & -0.04 \\
Average & & & & & 0.097 & -0.035 \\
$\sigma$ & & & & & 0.03 & 0.09 \\
\hline E3 & 6390 & 6550 & 3.9 & 2.1 & 0.27 & 0.16 \\
E56 & 6360 & 6500 & 3.9 & 2.1 & 0.11 & -0.06 \\
E19 & 6127 & 6500 & 3.9 & 2.1 & 0.14 & 0.00 \\
E99 & 6656 & 6800 & 4.0 & 2.0 & $(0.32)$ & $(0.23)$ \\
E17 & 6653 & & & & & \\
E25 & 6514 & 6900 & 4.1 & 1.9 & 0.11 & 0.06 \\
E5 & 6750 & 7100 & 4.3 & 1.9 & $(0.40)$ & $(0.22)$ \\
T1228 & 6600 & 6800 & 4.2 & 1.7 & 0.26 & 0.19 \\
E14 & 6690 & 6800 & 4.3 & 1.9 & 0.20 & 0.14 \\
E34 & 6480 & 6850 & 4.3 & 1.9 & $(0.45)$ & $(0.45)$ \\
E15 & 6380 & 6850 & 4.4 & 1.8 & 0.21 & 0.07 \\
E79 & 6460 & 6650 & 4.3 & 1.7 & 0.17 & 0.14 \\
Average & & & & & 0.18 & 0.09 \\
$\sigma$ & & & & & 0.06 & 0.08 \\
\hline *64 & 6390 & 6650 & 4.3 & 1.7 & 0.09 & -0.05 \\
E86 & 6300 & 6600 & 4.3 & 1.7 & 0.13 & 0.06 \\
T2105 & 5990 & 6350 & 4.3 & 1.1 & 0.04 & -0.05 \\
T2105 & 5990 & 6300 & 4.3 & 1.1 & 0.07 & 0.02 \\
T2105 & 5990 & 6400 & 4.4 & 1.1 & 0.13 & 0.05 \\
E7 & 6150 & 6300 & 4.3 & 1.1 & 0.13 & 0.12 \\
E7 & 6150 & 6300 & 4.3 & 1.1 & 0.12 & 0.07 \\
E45 & 5960 & 6350 & 4.3 & 1.1 & 0.12 & 0.08 \\
E45 & 5960 & 6350 & 4.3 & 1.1 & 0.13 & 0.03 \\
Average & & & & & 0.107 & 0.04 \\
$\sigma$ & & & & & 0.03 & 0.06 \\
\hline & & & & & & \\
\hline
\end{tabular}

for the IC 4651 stars. For the Sun we used an Edvardsson et al. model with $T_{\text {eff }}=5750 \mathrm{~K}, \log g=4.4$ and $\xi=0.9 \mathrm{~km} \mathrm{~s}^{-1}$. We note that our solar Fe I and Fe II abundances are slightly higher than the canonical value $(\log n(\mathrm{Fe})=7.52$, Anders \& Grevesse 1989). In particular, it is worth noting that while in the Sun the ionization equilibrium is obtained, this is not the case for most stars in IC 4651; for these Fe II is usually lower than Fe I because we allowed the spectroscopic $\log g$ to depart no more than 0.3 dex from the photometric value.

The typical $1 \sigma$ dispersion around the mean $\mathrm{Fe} \mathrm{I}$ and Fe II abundances is 0.1 dex, very uniform between slowrotating dwarfs and giants.

Table 2 shows that:

1. the abundances obtained from different spectra of the same star (7 last entries in the table) are in very good agreement, within 0.09 dex for Fe I and 0.1 dex for Fe II (peak to peak);
Table 3. Photometric $T_{\text {eff }}$ and associated $[\mathrm{Fe} / \mathrm{H}]$ values for the rapidly rotating TO stars in IC 4651, computed with our revised reddening estimate $(E(b-y)=0.091)$.

\begin{tabular}{lccccr}
\hline \hline Star & $T_{\text {phot }}$ & $\log g$ & $\xi$ & {$[\mathrm{Fe}$ I $]$} & {$[\mathrm{Fe}$ II $]$} \\
\hline E3 & 6550 & 3.9 & 2.1 & 0.27 & 0.16 \\
E56 & 6520 & 3.9 & 2.1 & 0.11 & -0.06 \\
E19 & 6280 & 3.9 & 2.1 & 0.02 & 0.02 \\
E99 & 6830 & 4.0 & 2.0 & 0.33 & 0.23 \\
E25 & 6680 & 4.0 & 2.0 & 0.00 & -0.02 \\
E5 & 6930 & 4.2 & 2.0 & 0.28 & 0.15 \\
T1228 & 6770 & 4.2 & 1.7 & 0.26 & 0.19 \\
E14 & 6860 & 4.2 & 1.9 & 0.23 & 0.18 \\
E34 & 6640 & 4.2 & 1.9 & 0.39 & 0.33 \\
E15 & 6540 & 4.2 & 1.7 & 0.08 & 0.06 \\
E79 & 6620 & 4.3 & 1.7 & 0.17 & 0.14 \\
\hline Average & & & & 0.15 & 0.13 \\
$\sigma$ & & & & 0.1 & 0.1 \\
\hline
\end{tabular}

2. in most cases spectroscopic temperatures are higher than photometric ones. While the difference between $T_{\text {eff }}$ (phot) and $T_{\text {eff }}$ (spec) is within $\sim 150 \mathrm{~K}$ for the giants, it can be as high as $300-400 \mathrm{~K}$ for the other stars. Small discrepancies could easily be explained, for instance by the characteristics of the stellar model atmospheres adopted, but the differences in $T_{\text {eff }}$ are too high for most dwarfs to be explained in this way;

3. the bulk of the stars $(19$, including all the giants) have Fe I ranging between $[\mathrm{Fe} / \mathrm{H}]=0.03$ and $[\mathrm{Fe} / \mathrm{H}]=0.26$, while the remaining three objects, all located at the $\mathrm{TO}$, give more than $2 \sigma$ higher Fe I abundances;

4. the average $\mathrm{Fe}$ I abundances of giants and dwarfs are in excellent agreement, while the average abundance of stars at the TO is somewhat higher, even when not taking into account the three stars mentioned above. More specifically, the six giants have a mean $[\mathrm{Fe} \mathrm{I} / \mathrm{H}]=0.10$, to be compared with $[\mathrm{Fe} \mathrm{I} / \mathrm{H}]=0.11$ for the solar-type stars. This is a very encouraging result, confirming that not only the spectroscopic effective temperatures are consistent, but also that the agreement between the two sets of model atmospheres is very good.

However, eight stars, which are typically rather fastrotating TO stars, give a slightly larger metallicity $([\mathrm{Fe} \mathrm{I} / \mathrm{H}]=0.18)$, while the three apparently most metalrich stars (all hot and very fast rotators) give $[\mathrm{Fe} \mathrm{I} / \mathrm{H}]$ above 0.3 (E5, E34, E99). Anticipating the conclusions of our discussion, we note that the abundances for all rapid rotators suffer substantial uncertainties, regardless whether their abundances agree with the other stars of the cluster. In addition, for TO stars our method is not sensitive enough to constrain $T_{\text {eff }}$ properly, and we shall see that, when adopting photometric $T_{\text {eff }}$ with proper reddening, we recover $\mathrm{Fe}$ abundances in very good agreement with the solar-type stars and the giants.

In the following, we discuss: a) possible reasons for the overabundance measured among rapidly rotating stars at the TO; 


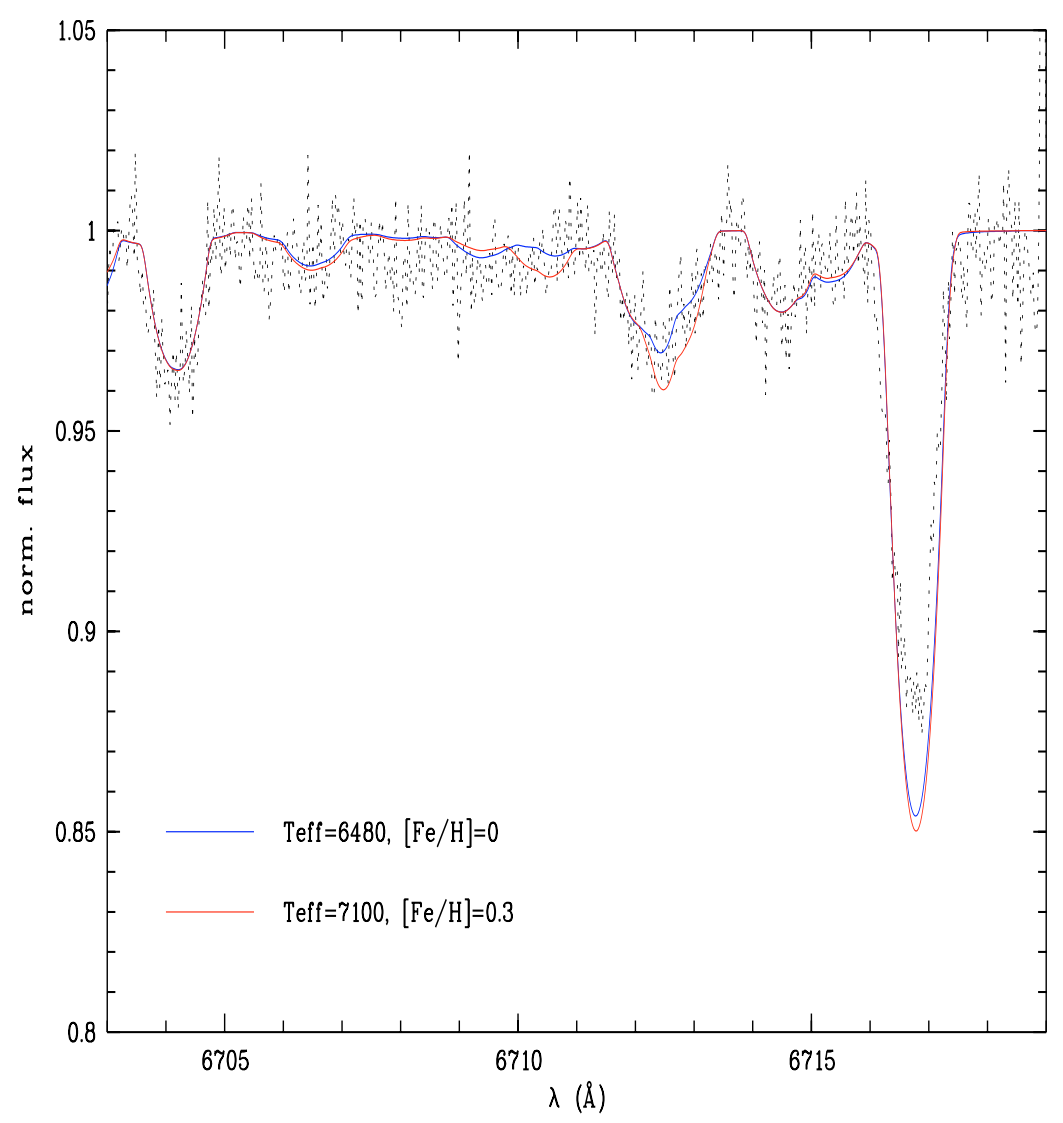

Fig. 2. Two synthetic spectra for the Li region in the fast-rotating E34, differing by $\sim 600 \mathrm{~K}$ in $T_{\text {eff }}$ and 0.3 in $[\mathrm{Fe} / \mathrm{H}]$; the observed spectrum cannot discriminate between these two solutions.

and $b$ ) possible reasons for the difference between spectroscopic and photometric $T_{\text {eff }}$ values.

\subsubsection{Hot fast-rotating turnoff stars}

Rapid rotation is a concern for abundance analyses based on equivalent widths, since, when the stars rotate fast, the selected lines may blend with nearby lines resulting in systematically higher equivalent widths. In addition, the measured scatter in $[\mathrm{Fe} / \mathrm{H}]$ for fast rotators $(\sigma \sim 0.3 \mathrm{dex})$ is about 3 times higher than for the best (slow rotating) stars in IC 4651. This large dispersion does not seriously degrade the formal uncertainties of the derived $\mathrm{Fe}$ abundance, because more than $60 \mathrm{Fe} \mathrm{I}$ lines were used in the determination. Rather, the problem is that by excluding many lines in the analysis we are no longer performing a strict solar comparison. In addition, the solution becomes quite insensitive to variations in $T_{\text {eff }}$, and the solution space becomes flat instead of showing a pronounced minimum.

We have confirmed this by artificially broadening the spectra of three stars (the Sun, E45 and E25) and repeating the analysis. We derive a spuriously large microturbulence and an $\mathrm{Fe}$ abundance up to 0.1 dex higher than from the original spectra. Both these points are consistent with the data in Table 2, which shows that indeed for virtually all TO stars the microturbulence velocity is higher than the canonical values, and that the (typically fast-rotating) $\mathrm{TO}$ stars have $[\mathrm{Fe} / \mathrm{H}]=$ 0.18 , i.e. about 0.1 dex higher than the mean value derived from the more slowly-rotating dwarfs and giants. However, the systematic effects due to higher rotation do not explain the large differences between spectroscopic and photometric temperatures.

Finally, a synthetic and rotationally broadened spectrum for star E34 around the Li I $6708 \AA$ doublet was computed using MOOG (Sneden 1973) and Kurucz (1995) model atmospheres. The observed spectrum of E34 is shown in Fig. 2, together with two different synthetic spectra. The figure clearly shows that we cannot discriminate between the two solutions, which differ by $600 \mathrm{~K}$ and $0.3 \mathrm{dex}$ in $[\mathrm{Fe} / \mathrm{H}]$.

Because of this uncertainty in the rapidly-rotating hot TO stars, we repeated the analysis using the photometric $T_{\text {eff }}$ derived with our best reddening estimate (cf. next section). The results are given in Table 3, where the $\mathrm{Fe}$ abundances of the $11 \mathrm{TO}$ stars have been redetermined with the new photometric temperatures. The scatter in the Fe abundances is quite large $(0.1 \mathrm{dex})$, but the mean $([\mathrm{Fe} \mathrm{I} / \mathrm{H}]=0.15)$ is now only slightly higher and in very good agreement with $[\mathrm{Fe} / \mathrm{H}]=0.10$ found for the giants and solar-type stars.

\subsubsection{Discrepancy between photometric and spectroscopic temperatures}

The average difference between spectroscopic and photometric $T_{\text {eff }}$ is 290 and $100 \mathrm{~K}$ for the slow-rotating dwarfs and the giants, respectively. There are three possible explanations 


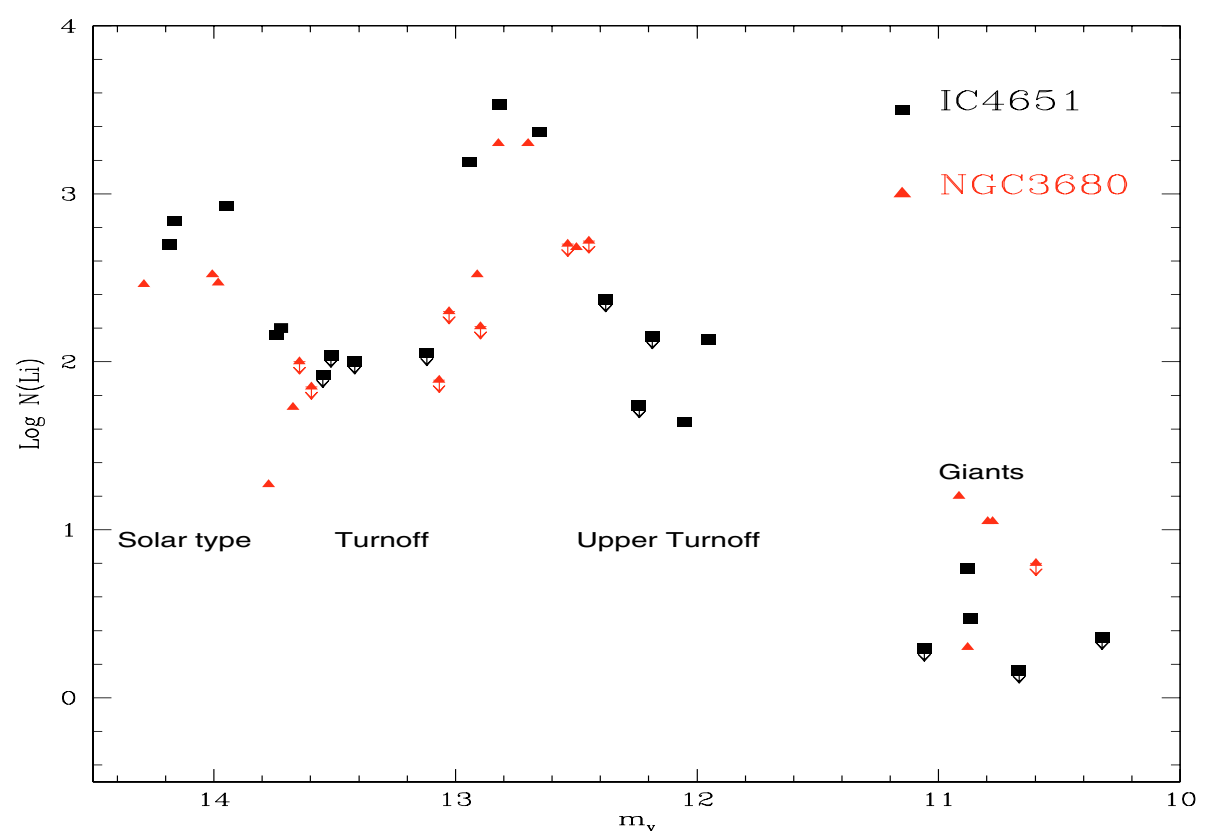

Fig. 3. Li abundance vs. $m_{V}$ for stars in IC 4651 (squares) and NGC 3680 (triangles).

for the difference: $i$ ) the use of an incorrect color- $T_{\text {eff }}$ calibration; ii) systematic effects due to the adopted analysis code and model atmospheres; and iii) an error in the estimate of reddening.

i) Using a different colour system and/or calibration, such as $B-V$ and the calibration of Soderblom et al. (1993), would not eliminate the discrepancy although, for some of the stars, it would result in differences of $\sim 200 \mathrm{~K}$ with respect to the temperatures from $b-y$ and the calibrations of Alonso et al. (1996, 1999). For example, Randich et al. (2002) estimated $T_{\text {eff }}$ of 6061,6016 , and $6110 \mathrm{~K}$ for E7, $\mathrm{E} 45$, and T2105, still 250-300 K below our spectroscopic temperatures.

Thus, problems related to the $(b-y)$ photometric scale are unlikely to be responsible for the discrepancy. We also point out that the spectroscopic temperatures of the main sequence stars cannot be strongly in error, given the excellent agreement in the $[\mathrm{Fe} / \mathrm{H}]$ between giants and dwarfs; were the $T_{\text {eff }}$ from photometry truly too low by $250 \mathrm{~K}$, the dwarfs would have subsolar $[\mathrm{Fe} / \mathrm{H}]$ abundances $([\mathrm{Fe} / \mathrm{H}] \sim$ $-0.2)$, in conflict with the giants and with the metallicity estimated from photometry.

ii) One could argue that the model atmospheres could be partly responsible for the discrepancy; in order to check this possibility, we have analyzed the Sun and E45 with MOOG (Sneden 1973) and the Kurucz (1995) model atmospheres. We find a dependence of the difference in the derived abundances (ours-MOOG) on both the line excitation potential and EWs, due to the fact that Kurucz models are somewhat warmer and that damping is treated differently in the two codes; however, for the Sun we find an Fe abundance only 0.03 dex higher $\left(\log n\left(\mathrm{Fe}_{\mathrm{I}}\right)=7.60\right)$, and for $\mathrm{E} 45[\mathrm{Fe} / \mathrm{H}]$ is exactly the same as found by us (0.15). The $\xi$ values agree within $0.2 \mathrm{~km} \mathrm{~s}^{-1}$ and $T_{\text {eff }}$ within $\sim 50 \mathrm{~K}$. The use of different model atmospheres and codes therefore does not introduce substantial differences in our results.

iii) We are therefore only left with the possibility that the interstellar excess estimated from photometry is too low. By adding 0.02 mag to the $E(b-y)$ by Meibom, the two $T_{\text {eff }}$ are brought almost into agreement. We conclude that $E(b-y)=$ 0.091 or slightly larger for IC 4651. Note that such an increase in the reddening would not degrade the already acceptable agreement between spectroscopic and photometric temperatures for the giants, since their temperature scale is less sensitive than that of the dwarfs to $b-y$ in this regime: with $E(b-y)=0.091$, the spectroscopic and photometric temperatures of the six giants agree within $5 \mathrm{~K}$.

Based on the above considerations and the more reliable and consistent abundances of the slowly-rotating giants and main sequence stars, we conclude that the Fe abundance of IC 4651 is $[\mathrm{Fe} / \mathrm{H}]=+0.10 \pm 0.03$. Using our higher value of $E(b-y)$ would increase the photometric estimate of $[\mathrm{Fe} / \mathrm{H}]$ by Meibom et al. (2002) by about 0.08 dex, still within the errors of both methods. The change in distance modulus is also within the errors and without consequence for our discussion.

\section{Other elements}

Individual element abundances for the stars in IC 4651, computed with the stellar parameters from the Fe analysis (Table 2), are given in Table 5. Table 6 lists mean abundances, element by element, for the Sun (both the canonical values from Anders \& Grevesse 1989, and our results), the main-sequence stars, and the giants in IC 4651. When computing the mean abundance for the giants we excluded E95, since for the discrepant elements (such as $\mathrm{Na}$ ), this star shares the abundance pattern of the dwarfs rather than the giants. The abundances we derive from the UVES solar spectrum are all within 0.1 dex of the 
canonical solar values, and we use them as the reference for the cluster stars.

We have checked the sensitivity of our abundances to the assumed stellar parameters by analyzing a giant (E60) and a dwarf star (E86) while varying $T_{\text {eff }}, \log g$, and $\xi$ separately. The results are given in Table 4.

\subsection{Comments on abundances}

Titanium and scandium: The Ti II and ScII abundances do not follow that of $\mathrm{Fe}$ II; $\mathrm{Fe}$ II appears to be underabundant in giants with respect to dwarfs (see Table 2), but the opposite is true for Ti II and ScII. While only two lines are available for ScII, the number of lines of are comparable for $\mathrm{Ti}$ II and $\mathrm{Fe}$ II, so there seems to be no simple explanation for this difference. If some overionization was present, we would indeed expect the ionized species to be more abundant in giants than in dwarfs, but this should be true for $\mathrm{Fe}$ II as well. A possible cause might be the strong dependence of $\mathrm{Fe}$ II on $T_{\text {eff }}$ and $\xi$ in the giants, as emerging from the error analysis of Table 4 . Since Ti II is much less sensitive to the atmospheric parameters other than gravity, some small mismatch in these may be enough to cause the different behaviour.

Magnesium: For $\mathrm{Mg}$ we only used the two lines at 6318.72 and $6319.24 \AA$.

Sodium: Na shows the largest difference between giants and dwarfs. In order to convince ourselves of the reality of this discrepancy, we have first checked how other effects, such as non-LTE, may affect our abundance computation. Gratton et al. (1999) published NLTE calculations for dwarfs and giants and showed that a small correction, of order $0.07 \mathrm{dex}$, is required for giants with $\log g \sim 3$, such as those analysed here. A similar correction applies to the dwarfs, however, so NLTE is not the cause of the discrepancy. Our analysis of the effects of changing the stellar parameters shows that the only way to reduce this difference would be to lower the temperature scale for the giants by about $200 \mathrm{~K}$. While temperatures $50 \mathrm{~K}$ cooler would help to bring the $\mathrm{Fe}$ ionization in balance in giants, values much larger would cause produce large discrepancies between dwarfs and giants in other elements, notably $\mathrm{Ca}$, Ti, and $\mathrm{Fe}$. We consider this possibility extremely unlikely and tentatively conclude that the difference in $\mathrm{Na}$ between giants and dwarfs is real, at the level of $\sim 0.2$ dex. We note that the only subgiant in the sample, E95, has a $\mathrm{Na}$ abundance compatible with that of the dwarfs.

Aluminium: $\mathrm{Al}$ is stronger in giants than in dwarfs; the discrepancy is marginally within the errors, however. NLTE effects must also be taken into account for Al: according to Baumüller \& Gehren (1997), NLTE effects should increase the Na abundance in stars near solar metallicity when going from the main sequence to $\log g=3$; they would therefore make the discrepancy even larger.

Chromium: In three stars (all dwarfs) we could measure only the line at $6362.88 \AA$. In the similar star T2105, this line gives an abundance about 0.2 dex higher than the other line used, at $6330.10 \AA$. The Cr I abundances given for these stars may
Table 4. Abundance changes $(\Delta[\mathrm{X} / \mathrm{H}])$ in two stars (E60 giant, E86 main sequence) in response to variations of $T_{\text {eff }}, \log g$, and $\xi$ ).

\begin{tabular}{lllll}
\hline \hline El. & $T_{\text {eff }}-100$ & $T_{\text {eff }}-200$ & $\log g-0.3$ & $\xi-0.3$ \\
\hline Al I & -0.06 & -0.13 & 0.01 & 0.05 \\
Ca I & -0.10 & -0.21 & 0.02 & 0.12 \\
Cr I & -0.15 & -0.32 & -0.02 & 0.22 \\
Fe I & -0.05 & -0.08 & -0.03 & 0.13 \\
Fe II & 0.13 & 0.27 & -0.16 & 0.11 \\
Mg I & -0.03 & -0.04 & -0.01 & 0.02 \\
Na I & -0.08 & -0.17 & 0.01 & 0.08 \\
Ni I & -0.03 & -0.04 & -0.06 & 0.17 \\
Si I & 0.05 & 0.10 & -0.04 & 0.06 \\
Ti I & -0.15 & -0.30 & -0.01 & 0.14 \\
Ti II & 0.04 & 0.09 & -0.13 & 0.05 \\
\hline & & E86 & & \\
Al I & -0.03 & -0.07 & 0.01 & 0.01 \\
Ca I & -0.03 & -0.07 & 0.01 & 0.02 \\
Fe I & -0.05 & -0.12 & +0.01 & 0.05 \\
Fe II & 0.01 & 0.02 & -0.09 & 0.08 \\
Mg I & -0.03 & -0.07 & 0.01 & 0.01 \\
Na I & -0.03 & -0.07 & 0.01 & 0.01 \\
Ni I & 0.06 & -0.12 & 0.00 & 0.03 \\
Si I & -0.02 & -0.06 & 0.03 & 0.03 \\
Ti I & -0.08 & -0.16 & 0.01 & 0.01 \\
Ti II & -0.01 & -0.02 & -0.20 & 0.08 \\
\hline & & & &
\end{tabular}

therefore be about 0.2 dex higher than the real values and have not been used in the final computation.

Lithium: Li deserves particular attention, since the abundance is computed from only one line, and $\mathrm{Li}$ is expected to vary along the CMD. $[\mathrm{Li} / \mathrm{H}]$ has been determined from the $6708 \AA$ resonance doublet using MOOG; the equivalent width for this line was measured both automatically and manually for all the stars. In some cases, differences as large as $15 \mathrm{~m} \AA$ between the two measurements were found; in those cases, the manual measurements were repeated and found to be more reliable than the automatic ones; this is explained by the fact that the Li doublet is broader than normal single lines, so our version of DAOSPEC likely considered the Li line as a blend and underestimated its equivalent width.

$\mathrm{S} / \mathrm{N}$ ratio estimates indicate upper limits to the measured equivalent widths as small as 1-2 $\mathrm{m} \AA$ in sharp-lined stars, but, because most of our stars rotate quite fast, we adopted conservative upper limits of $5 \mathrm{~m} \AA$. For rapid rotators the measured equivalent width includes the contribution of the Fe 6707.46 line; for these stars, the measured EWs were corrected using the analytical approximation by Soderblom et al. (1993). $B-V$ colors were corrected for reddening using our revised estimate of $E(b-y)=0.091$, corresponding to $E(B-V)=$ 0.13 .

The $\mathrm{Li}$ abundance is very sensitive to the adopted $T_{\text {eff }}$; we have computed it using both the spectroscopic $T_{\text {eff }}$ from the 
Table 5. Element abundances for individual solar-type dwarfs and giants in IC 4651. Entries with “:” refer to uncertain measurements. For Cr, see text.

\begin{tabular}{|c|c|c|c|c|c|c|c|c|c|c|c|c|c|c|c|}
\hline Elem. & E45 & E45 & E7 & E7 & $\mathrm{T} 2105$ & T2105 & E86 & E64 & E25 & E12 & E98 & E60 & E812 & E95 & E8 \\
\hline$[\mathrm{Na} \mathrm{I} / \mathrm{H}]$ & 0.02 & 0.06 & 0.14 & 0.11 & -0.10 & -0.03 & $-0.18:$ & 0.10 & 0.08 & 0.34 & 0.30 & 0.28 & 0.35 & 0.03 & 0.27 \\
\hline$[\mathrm{Mg} \mathrm{I} / \mathrm{H}]$ & 0.12 & 0.17 & 0.18 & 0.19 & 0.24 & 0.25 & 0.25 & 0.22 & 0.24 & 0.17 & 0.22 & 0.18 & 0.21 & 0.31: & 0.18 \\
\hline [Al I/H] & 0.00 & 0.01 & 0.08 & 0.08 & 0.03 & 0.05 & 0.11 & -0.01 & 0.08 & 0.18 & 0.24 & 0.13 & 0.19 & 0.10 & 0.19 \\
\hline$[\mathrm{Si} \mathrm{I} / \mathrm{H}]$ & 0.17 & 0.18 & 0.23 & 0.19 & 0.06 & 0.07 & 0.27 & 0.24 & 0.25 & 0.15 & 0.21 & 0.20 & 0.19 & 0.23 & 0.14 \\
\hline$[\mathrm{Ca} \mathrm{I} / \mathrm{H}]$ & 0.15 & 0.15 & 0.15 & 0.15 & 0.12 & 0.18 & 0.19 & 0.14 & 0.21 & 0.12 & 0.11 & 0.04 & 0.09 & 0.17 & 0.09 \\
\hline$[\mathrm{Sc} \mathrm{II} / \mathrm{H}]$ & -0.04 & -0.02 & -0.06 & -0.09 & -0.26 & -0.21 & -0.19 & 0.04 & 0.05 & 0.03 & 0.19 & 0.15 & 0.07 & -0.10 & 0.02 \\
\hline [Ti I/H] & 0.14 & 0.16 & 0.19 & 0.14 & 0.25 & 0.27 & 0.18 & 0.35: & 0.33: & 0.17 & 0.25 & 0.24 & 0.20 & 0.10 & 0.23 \\
\hline [Ti II/H] & -0.07 & -0.06 & 0.01 & 0.01 & -0.08 & -0.06 & 0.13 & 0.16 & 0.26 : & 0.04 & 0.23 & 0.19 & 0.16 & 0.11 & 0.10 \\
\hline$[\mathrm{Ni} \mathrm{I} / \mathrm{H}]$ & 0.09 & 0.15 & 0.13 & 0.16 & 0.03 & 0.07 & 0.09 & 0.16 & 0.20 & 0.14 & 0.28 & 0.24 & 0.15 & 0.05 & 0.20 \\
\hline$[\mathrm{Cr} \mathrm{I} / \mathrm{H}]$ & 0.18 & 0.20 & 0.26 & 0.20 & 0.18 & 0.29 & 0.47 : & 0.37 : & 0.71: & 0.08 & 0.12 & 0.11 & 0.01 & -0.17 & 0.08 \\
\hline
\end{tabular}

Table 6. Average element abundances in the sun and in IC 4651, separately for the dwarfs and giants; numbers in parenthesis gives the average number of lines used in the mean. [Ti II/Fe] is relative to the solar Fe II abundance.

\begin{tabular}{|c|c|c|c|c|c|c|c|}
\hline \multirow[t]{2}{*}{ Elem. } & \multicolumn{2}{|c|}{ Sun } & \multirow[t]{2}{*}[\mathrm{X}/\mathrm{H}]{$_{\mathrm{MS}}$} & \multirow[t]{2}{*}[\mathrm{X}/\mathrm{H}]{$_{\text {giants }}$} & \multirow[t]{2}{*}[\mathrm{X}/\mathrm{H}]{$_{\text {Cluster }}$} & \multirow[t]{2}{*}[\mathrm{X}/\mathrm{Fe}]{$_{\mathrm{MS}}$} & \multirow[t]{2}{*}[\mathrm{X}/\mathrm{Fe}]{$_{\text {giants }}$} \\
\hline & $A$ and $G$ & Us & & & & & \\
\hline $\mathrm{Fe}_{\mathrm{I}}(100)$ & 7.52 & 7.57 & 0.11 & 0.10 & 0.11 & & \\
\hline $\mathrm{Fe}_{\text {II }}(7)$ & 7.52 & 7.59 & 0.02 & -0.04 & / & & \\
\hline $\mathrm{Na}$ I (2) & 6.33 & 6.28 & 0.02 & 0.29 & I & -0.09 & 0.19 \\
\hline $\operatorname{Mg}_{\text {I }}(2)$ & 7.58 & 7.53 & 0.24 & 0.19 & 0.21 & 0.13 & 0.09 \\
\hline Al I (2) & 6.47 & 6.45 & 0.04 & 0.17 & 0.11: & -0.07 & 0.07 \\
\hline Si I (5) & 7.55 & 7.45 & 0.18 & 0.18 & 0.18 & 0.07 & 0.08 \\
\hline Ca I (17) & 6.36 & 6.38 & 0.15 & 0.10 & 0.13 & 0.04 & 0.00 \\
\hline Sc II (2) & 3.10 & 3.14 & -0.09 & 0.09 & 0.0 : & -0.11 & 0.11 \\
\hline Ti I (21) & 4.99 & 4.99 & 0.19 & 0.22 & 0.20 & 0.08 & 0.12 \\
\hline Ti II (5) & 4.99 & 4.88 & 0.02 & 0.14 & 0.08 & 0.00 & 0.18 \\
\hline Ni I (15) & 6.25 & 6.23 & 0.12 & 0.20 & 0.16 & 0.01 & 0.10 \\
\hline Cr I (2) & 5.67 & 5.72 & 0.22 & 0.08 & 0.15 & 0.11 & -0.02 \\
\hline
\end{tabular}

Fe analysis and the photometric $T_{\text {eff }}$ using the Alonso scale and $E(b-y)=0.091$ (see above). For most stars the difference is negligible, and in no case will it influence our conclusions. We estimate the uncertainty in $T_{\text {eff }}$ to be about $200 \mathrm{~K}$ for dwarfs and TO stars and half that amount for the giants, corresponding to typical uncertainty of $0.2 \mathrm{dex}$ in $[\mathrm{Li} / \mathrm{H}]$ for all the stars. However, most of this is due to the uncertainty in $E(b-y)$, and a change in $E(b-y)$ would produce an almost constant shift of the Li abundances without affecting most of our conclusions, which are based on the pattern of changes in $\mathrm{Li}$ along the CMD.

Table 7 reports the Li equivalent widths and abundances for all the stars. Asterisks denote stars with unblended Li lines; for the other stars, the equivalent widths include the Fe I blend.

\section{Discussion}

\subsection{Chemical composition of IC 4651}

Our mean abundances for $\mathrm{Fe}$ and several other elements in IC 4651 are all reported in Table 6. For most elements, accurate mean abundances for the whole cluster can be derived, given the very good agreement between the results for individual stars. In the following we will use the results for disk stars by Edvardsson et al. (1993) and Chen et al. (2000) as our reference in the comparisons.

\subsection{1. $\alpha$ elements: $\mathrm{Mg}, \mathrm{Si}, \mathrm{Ti}$ and $\mathrm{Ca}$}

$[\mathrm{Mg} / \mathrm{Fe}]$ is the same for giants and dwarfs and slightly above Solar $([\mathrm{Mg} / \mathrm{Fe}]=0.11)$. Considering that the cluster is metal rich, this fits the general trend of $[\mathrm{Mg} / \mathrm{Fe}]$ in disk stars extremely well. In their discussion, Chen et al. (2000) conclude that the mechanisms of $\mathrm{Mg}$ production are not well understood, as some $\mathrm{Mg}$ seems to be produced much after SNe II cease to dominate the enrichment.

$[\mathrm{Si} / \mathrm{Fe}]$ and $[\mathrm{Ti} / \mathrm{Fe}]$ are also very well defined and slightly positive (0.07-0.08), like Mg. Together, the slight overabundance of all these elements is highly significant and fits the trend in field stars of comparable metallicity. In contrast, $[\mathrm{Ca} / \mathrm{Fe}]$ is not significantly different from zero, so $\mathrm{Ca}$ seems not to share the overabundance of the other $\alpha$-elements. 
Table 7. Lithium abundances for the stars in IC 4651, computed with both spectroscopic (S) and photometric (P) values of $T_{\text {eff }}$, assuming a reddening of $E(b-y)=0.091$; we use the latter in our analysis. Asterisks indicate that $W(\mathrm{Li})$ (in $\mathrm{m} \AA$ ) refers to the unblended $\mathrm{Li}$ line.

\begin{tabular}{lrrrrr}
\hline \hline Star & $T(\mathrm{~S})$ & $T(\mathrm{P})$ & $W(\mathrm{Li})$ & $\log N(\mathrm{Li})(\mathrm{S})$ & $\log N(\mathrm{Li})(\mathrm{P})$ \\
\hline E12 & 5000 & 5020 & $<5$ & $<0.35$ & $<0.38$ \\
E8 & 4900 & 4950 & $<5$ & $<0.09$ & $<0.23$ \\
E98 & 4900 & 4950 & $16 *$ & 0.72 & 0.82 \\
E60 & 4900 & 4930 & $9 *$ & 0.42 & 0.53 \\
E812 & 5000 & 4910 & $<5$ & $<0.38$ & $<0.21$ \\
E95 & 5800 & 5670 & $35 *$ & 2.19 & 2.07 \\
E3 & 6550 & 6550 & 9 & 1.64 & 1.64 \\
E56 & 6500 & 6520 & $<10$ & $<2.15$ & $<2.16$ \\
E19 & 6500 & 6280 & $<5$ & $<1.83$ & $<1.66$ \\
E99 & 6800 & 6830 & $<10$ & $<2.36$ & $<2.38$ \\
E25 & 6900 & 6680 & 75 & 3.44 & 3.29 \\
E5 & 7100 & 6930 & 76 & 3.59 & 3.47 \\
T1228 & 6800 & 6770 & 58 & 3.20 & 3.18 \\
E14 & 6800 & 6860 & $<5$ & $<2.03$ & $<2.07$ \\
E34 & 6850 & 6640 & $<5$ & $<2.07$ & $<1.92$ \\
E15 & 6850 & 6540 & $<6$ & $<2.15$ & $<1.93$ \\
E79 & 6650 & 6620 & $<5$ & $<1.93$ & $<1.91$ \\
E64 & 6650 & 6550 & $10 *$ & 2.24 & 2.17 \\
E86 & 6600 & 6459 & $10 *$ & 2.20 & 2.13 \\
T2105 & 6350 & 6138 & $67 *$ & 3.02 & 2.84 \\
E7 & 6300 & 6308 & $53 *$ & 2.83 & 2.84 \\
E45 & 6350 & 6103 & $47 *$ & 2.80 & 2.59 \\
\hline & & & & &
\end{tabular}

Together, our results strongly indicate that none of the $\alpha$-elements is underabundant at this metallicity, so that the $[\alpha / \mathrm{Fe}]$ relation flattens out, or even increases, at high metallicity.

\subsubsection{Sodium and aluminium}

The behaviour of $\mathrm{Na}$ and $\mathrm{Al}$ is unfortunately less clean, mostly because of the abundance differences between giants and dwarfs. Our dwarf data would indicate that Al is slightly underabundant, at odds with what is found among field stars (Chen et al. 2000), while the low value of $[\mathrm{Na} / \mathrm{Fe}]$ would completely agree with the field data, which are flat and scattered above the Solar $[\mathrm{Fe} / \mathrm{H}]$. We note also that low $\mathrm{Na}$ and $\mathrm{Al}$ are observed for the young stars in the Chen sample, which would be in better agreement with our data for the relatively young IC 4651. Given the discrepancy between giants and dwarfs, we can only conclude that the ratio of these elements to $\mathrm{Fe}$ is solar or slightly below for IC 4651 .

For $\mathrm{Na}$, we note that the dwarfs and the low-luminosity giant of the sample (E95) share the same low Na value, while all the other giants have much higher $\mathrm{Na}$. We believe that this $[\mathrm{Na} / \mathrm{Fe}]$ enhancement in the giants with respect to the dwarfs is real. A possible Na overabundance of the order of 0.2 dex has been identified also by Tautvaisiene et al. (2000) among the clump giants of M 67, very similar to what we find in IC 4651
(0.28 dex). On the other hand, the same authors found no $\mathrm{O}$ deficiency nor any $\mathrm{Al}$ overabundance in their stars, showing that if the Na overabundance is due to internal nucleosynthesis plus mixing, then this process is not accompanied by the $\mathrm{MgAl}$ cycle. This would agree with other studies, which have shown that the $\mathrm{MgAl}$ cycle dominates at lower metallicity.

Na overabundances have been observed in A-F supergiants (Takeda \& Takeda-Hiday 1994, and references therein). They are explained simply by a first-dredge up of ${ }^{23} \mathrm{Na}$, synthesized by the Ne-Na mode of H-burning while the star was on the main sequence (El Eid \& Champagne 1995). Theory predicts that the post dredge-up enhancement of $\mathrm{Na}$ should increase with stellar mass so much less ${ }^{23} \mathrm{Na}$ should be synthesized inside the present giant stars in IC 4651 than in these A-F supergiants: Our $3.0 M_{\odot}$ and $2.2 M_{\odot}$ models (see Sect. 6.2.2) predict a $\mathrm{Na}$ enhancement of $0.3 \mathrm{dex}$ and $0.12 \mathrm{dex}$, respectively, with respect to the initial main-sequence value.

Given the substantial uncertainty of the nuclear reactions involved in the ${ }^{23} \mathrm{Na}$ production (Arnould et al. 1999) as well as the errors of our $\mathrm{Na}$ abundance determinations ( $0.15 \mathrm{dex}$ for a $T_{\text {eff }}$ error of $100 \mathrm{~K}$ in the giants), we see no serious conflict between first dredge-up predictions and the observed $\mathrm{Na}$ abundances in the giant stars. We note, however, that if the reported difference in M 67 is confirmed, this interpretation might be more problematic, given the substantially lower TO mass of the older M 67.

\subsubsection{The Fe group: $\mathrm{Cr}$ and $\mathrm{Ni}$}

With $[\mathrm{Ni} / \mathrm{Fe}]=[\mathrm{Cr} / \mathrm{Fe}]=0.05$, both elements share the overabundance of $\mathrm{Fe}$ and the general trends found in field stars.

\subsection{Lithium}

\subsubsection{Li abundances along the cluster sequence}

In a detailed study of the intermediate-age open cluster NGC 3680, Pasquini et al. (2001) found, in addition to the standard features (i.e. the Li dip and the plateau for solar-type stars), that: a) Li reaches young Pop. I values on the bright side of the dip; $b$ ) Li drops dramatically in even brighter stars, as soon as they become slightly evolved; and c) Giants very close together in the CMD show substantial scatter in Li abundance, with the clump giants having a measurable amount of $\mathrm{Li}$.

Given its similar age to NGC 3680 (within 0.1-0.2 Gyr, Meibom et al. 2002), IC 4651 is a perfect object with which to test whether the results for NGC 3680 may apply to other clusters, and thus potentially to entire disk population of age $\sim 1.5-1.7$ Gyr.

In Fig. 3 we plot the $\mathrm{Li}$ abundance vs. $\mathrm{m}_{\mathrm{V}}$ magnitude for both clusters (which are also at the same distance). The Li data for IC 4651 are from Table 7 (based on the photometric $T_{\text {eff }}$ ), with an estimated uncertainty of $0.2 \mathrm{dex}$; the NGC 3680 data are from Pasquini et al. (2001). For comparison between the clusters this is preferable to the classical Li vs. $T_{\text {eff }}$ diagram because of the degeneracy of the latter in the critical turnoff region (Pasquini et al. 2001). 
The different sections of the CMD are also indicated in Fig. 3. Moving from left to right, the diagram thus shows what happens in stars with increasing initial mass.

The Li abundance is an extremely tight function of $m_{V}$, confirming that the pattern observed in NGC 3680 is indeed common to both clusters; viz.:

- Stars fainter than the Li dip (the 3 solar-type stars E7, E45 and T2105 analysed by Randich et al. 2000) have roughly the same Li abundance, slightly below the younger Hyades stars at the same temperature. Note that our values differ from those by Randich et al. (2000) because of the substantially higher $T_{\text {eff }}$ values adopted in the present work. The general discussion of solar-type stars in clusters in Pasquini (2000) and Randich et al. $(2000,2002)$ will not be repeated here.

- The Li dip is extremely very well defined, although for some of our stars we can only give upper limits to the $\mathrm{Li}$ abundance.

- Immediately above the dip, the slightly more luminous stars (E5, E25, and T1228) have the meteoritic Li abundance (e.g. the initial Li content of the solar system, $[\mathrm{Li} / \mathrm{H}] \sim 3.3$ ) or even higher. This is near the bluest point on the TO, before the redward "hook" in the isochrones. Given the uncertainties in $T_{\text {eff }}$, we do not consider the apparent supermeteoritic value to be significant. It is clear, however, that these stars have not suffered any significant surface depletion of Li during their main-sequence lifetime.

- In dramatic contrast, the Li abundance drops precipitously by a factor of 10 or more in the stars that are just 0.25 mag brighter (E99 and the slightly brighter E3, E19 and E56).

- Finally, the giants exhibit an extremely interesting behaviour: out of five stars, three have only upper limits while $\mathrm{Li}$ is clearly detected in the two others, which are located in the clump region of the CMD.

In the following subsection we return to these patterns, which appear to be signatures of non-standard mixing processes in the stars.

\subsubsection{Comparison with the predictions from rotating stellar models}

The study of $\mathrm{Li}$ in open clusters is a very powerful tool to investigate the dynamical phenomena in the stellar interiors (e.g., Pasquini 2000; Deliyannis et al. 2000; Randich et al. 2000). In Talon \& Charbonnel (1998, hereafter TC98) and Palacios et al. (2003, hereafter PTCF03), measurements of Li abundances and rotational velocities in young open clusters ( $\alpha$ Per, Pleiades, Ursa Major, Coma Berenices, and the Hyades) were used to test the predictions of rotating stellar models including the most recent theoretical developments concerning meridional circulation, turbulence by shear instability, and atomic diffusion (see the above papers for a detailed description of the treatment of rotational mixing).

TC98, in particular, showed that the hot side of the Li dip in these young clusters is well described by these models, which also successfully reproduce the $\mathrm{He}, \mathrm{C}$, and $\mathrm{N}$ anomalies in
O-type and early B-type stars (Talon et al. 1997) and in O supergiants, as well as the B depletion in main-sequence B-type stars (see Maeder \& Meynet 2000 for references). Charbonnel \& Talon (1999, hereafter CT99) and PTCF03 extended the comparison between model predictions and observations of $\mathrm{Li}$ to evolved stars originating from the hot side of the dip. At that time, however, the necessary data along the subgiant branch were available only for field stars (Wallerstein et al. 1994; Lèbre et al. 1999; do Nascimento et al. 1999; Randich et al. 1999), while the data for open clusters concerned only bright giants (Gilroy 1989). The comparison between the models and the data was very satisfactory.

Our present analysis, together with the previous results for NGC 3680, fills several gaps in the observational picture and provides tighter constraints on the models. On the one hand, we now have lithium data for main-sequence stars in open clusters with an age intermediate between the very well-studied young population (younger than the Hyades) and the old cluster M 67. On the other hand, our abundance determinations trace the behaviour of lithium along the subgiant branch more precisely. This allows us to test the rotating models as regards the agedependence of the Li dip and the Li post-main-sequence evolution simultaneously.

In the following we focus our attention on the stars on the blue side of the Li dip, ignoring the main-sequence stars cooler than $6500 \mathrm{~K}$. For these "solar-type stars" indeed it has already been shown (Pasquini et al. 1994; Randich et al. 2000, 2002) that current models do not match the Li and Be observations. TC98 discussed this problem in terms of an additional mechanism, possibly related to internal gravity waves, which transports angular momentum efficiently in these lower-mass stars while it is inefficient on the blue side of the dip (see also CT99 and TC03 for more details). New models for low-mass stars of the red side of the dip, including angular momentum transport by gravity waves in addition to the hydrodynamical processes induced by rotation, are not yet available.

Here, we compare the predictions of the CT99 and PTCF03 models for stars on the blue side of the dip with the Li data in IC 4651 in the classical way, i.e., as functions of $T_{\text {eff }}$ separately for the main-sequence and turnoff stars (Fig. 4) and for the evolved stars (Fig. 5). The small differences in the description of the rotation-induced mixing in the two sets of models (see PTCF03 for a detailed discussion) enable us to combine their predictions and compare them to the present data. The initial rotation velocity used in both studies was very similar (100 or $110 \mathrm{~km} \mathrm{~s}^{-1}$ ), and the stars were assumed to undergo magnetic braking leading to the observed rotation velocities at the age of the Hyades.

Main sequence and turnoff stars

Figure 4 compares the predictions of the models for an age of 1.5 Gyr to our observational data for the main-sequence and turnoff stars in IC 4651. Our photometric values of $T_{\text {eff }}$ and the corresponding $\log N(\mathrm{Li})$ are shown (black points: actual determinations; black triangles: upper limits). Open symbols show the model predictions at 1.5 Gyr. The open circles correspond to models with an initial rotation velocity of $110 \mathrm{~km} \mathrm{~s}^{-1}$. For the $1.5 M_{\odot}$ model, this circle is for an initial $V_{\text {rot }}$ of $100 \mathrm{~km} \mathrm{~s}^{-1}$, and it is connected to the predictions for an initial velocity 


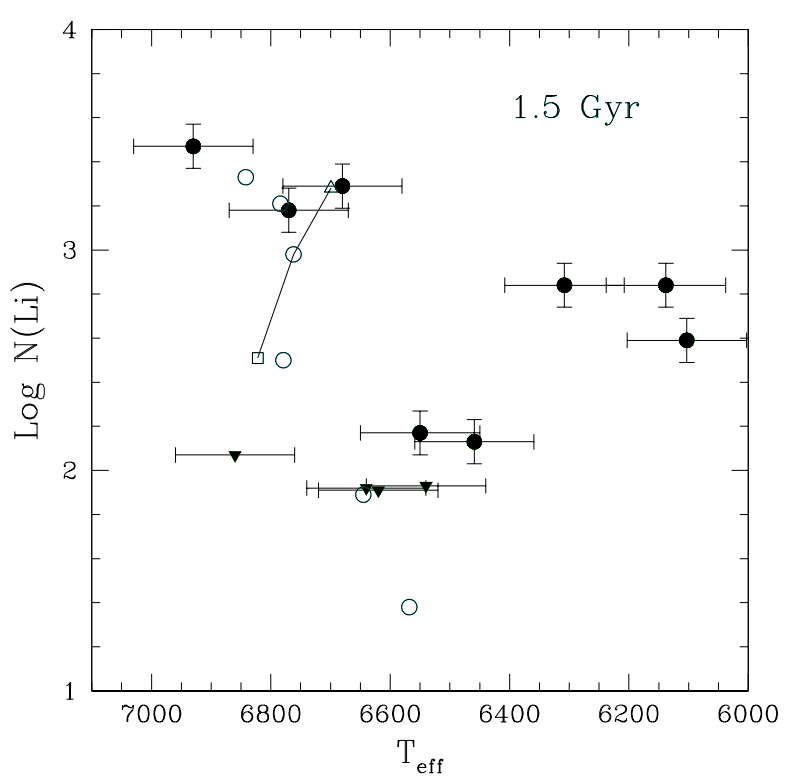

Fig. 4. Li abundance in main-sequence and turnoff stars fainter than $V \sim 12.6$ in IC 4651 (black points: actual determinations; black triangles: upper limits) vs. $T_{\text {eff }}$ from photometry. Open circles show model predictions from CT99 and PTCF03 for an initial $V_{\text {rot }}$ of $110 \mathrm{~km} \mathrm{~s}^{-1}$ and different masses. For $1.5 M_{\odot}$, additional models for an initial $V_{\text {rot }}$ of 50 and $150 \mathrm{~km} \mathrm{~s}^{-1}$ are also shown (open triangle and square, respectively).

of $50 \mathrm{~km} \mathrm{~s}^{-1}$ (open triangle) and $150 \mathrm{~km} \mathrm{~s}^{-1}$ (open square). These points provide an estimate of the expected dispersion at an age of $1.5 \mathrm{Gyr}$ for stars inside the Li dip which have undergone relatively strong braking early on the main sequence; it should be lower for more massive stars with thinner convective envelopes, for which the braking is less efficient. As can be seen, the models that already explained the shape of the hot side of the Li dip in the young open clusters also successfully account for the Li behaviour at later ages.

We note that the rotating models have been computed without adhoc convective core overshooting. However, rotational mixing naturally extends the convective core compared to the classical case, and has a similar effect as overshooting on the main-sequence lifetime and the turnoff shape in the HRD (this also explains why models for a given initial mass, but different initial rotational velocities show a range in $T_{\text {eff }}$ at a given age, as seen in Fig. 4 for the $1.5 M_{\odot}$ model). More extended grids of rotating models and corresponding isochrones now need to be computed and compared to colour-magnitude diagrams of open clusters with various ages.

\section{Subgiant stars}

We now turn to our sample of post-turnoff stars in IC 4651 . Figure 5 compares their $\mathrm{Li}$ abundances as a function of $T_{\text {eff }}$ with stellar models from PTCF03. The dashed lines show the evolution of the surface $\mathrm{Li}$ abundance in standard (i.e. non-rotating) models for $1.8 M_{\odot}$ and $2.2 M_{\odot}$. In both cases, surface Li depletion only begins inside the Herzsprung gap at $\sim 5800 \mathrm{~K}$ and is simply due to dilution as the convective zone deepens. The observed drop in lithium at much higher $T_{\text {eff }}$ cannot be explained by just this classical effect. Exactly the same behaviour is observed in NGC 3680 stars and in other clusters with turnoff

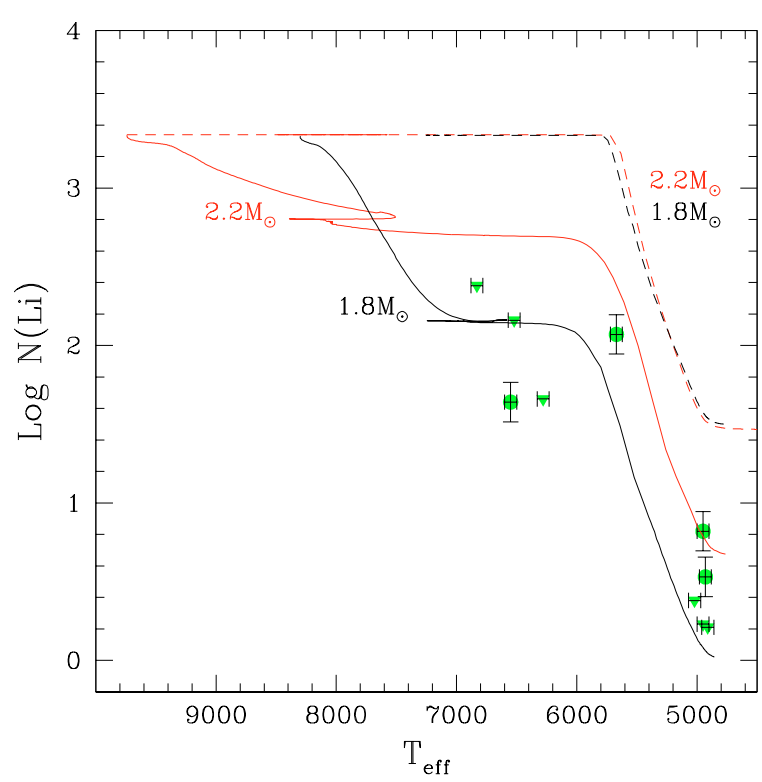

Fig. 5. Li abundances in post-turnoff stars brighter than $V \sim 12.6$ and giants in IC 4651 (photometric $T_{\text {eff }}$ values). The lines show the evolution of surface $\mathrm{Li}$ in standard models (dashed) and in two rotating models computed with an initial $V_{\text {rot }}$ of $110 \mathrm{~km} \mathrm{~s}^{-1}$ (PTCF03; full lines).

masses above $\sim 1.6 M_{\odot}$ (Gilroy 1989; Pasquini et al. 2001) and in field stars (see PTCF03 and references therein).

This points to a mechanism which is characteristic of stars originating from the hot side of the Li dip and is already efficient on the main sequence, even if its signature appears only as the stars start to cross the Hertzsprung gap (Vauclair 1991; Charbonnel \& Vauclair 1992). By enlarging the Li-free regions inside these objects relative to the classical case, rotationinduced mixing actually prepares the stars for the $\mathrm{Li}$ abundance variations seen in later evolutionary phases. The predicted behaviour of their surface Li is shown in Fig. 5 (full lines).

As can be seen, the rotating model for $1.8 M_{\odot}$ (the approximate turnoff mass of IC 4651) exhibits earlier surface Li depletion than in the classical case (i.e., at higher $T_{\text {eff }}$ ). This model, computed with an initial rotation velocity of $110 \mathrm{~km} \mathrm{~s}^{-1}$ as described previously, also explains the data for field subgiant stars, as discussed in CT99 and PTCF03 (see Fig. 10 of PTCF03). The present comparison shows that it also nicely matches the data for the subgiant stars in IC 4651. As already discussed for our main-sequence stars, one expects a dispersion in initial $V_{\text {rot }}$ which will account for the observed dispersion in the sample.

\section{Giant stars}

We end our discussion with the giant stars in IC 4651, which are also included in Fig. 5 (data points at 4900-5000 K). As indicated by isochrone fits to the cluster (see e.g. Meibom et al. 2002), these stars have masses higher than $1.8 M_{\odot}$ (the exact mass range depending on the physics included in the various sets of models). As can be seen, standard models for these objects lead to post-dilution $\mathrm{Li}$ abundances that are much higher than observed. The rotating models, however, predict a total Li depletion after dredge-up that is much larger than in the standard case, and again fit the observations very satisfactorily. 
The conclusions by CT99 and PTCF03, that the rotating models can explain the mean Li depletion and abundance dispersion in evolved stars above $\sim 1.4 M_{\odot}$ in the field and in open cluster are thus confirmed.

Cluster giants were discussed extensively by Pasquini et al. (2001), who speculated that clump giants could show more Li than stars on the first ascent of the RGB. Our observations of IC 4651 could possibly support this hypothesis. Indeed also in this cluster we observe only upper limits for the stars that are found away from the clump, which are lower than our typical detections in the stars in the clump region. Again, rotating models could provide a plausible explanation: in a given cluster, the clump giants had slightly higher initial masses than the RGB stars, and both theory and observations imply that they have undergone more modest braking while on the main sequence; thus, retaining more Li. This can be seen in Fig. 5, where the Li abundance after dredge-up in the $2.2 M_{\odot}$ rotating model is higher than in the $1.8 M_{\odot}$ model and fits the clump data perfectly. However, in a given cluster the difference in masses between RGB and clump stars can be at most of $0.1 M_{\odot}$, which does not seem enough to justify the difference.

The real point is that in the considered clusters it is very difficult to firmly establish which really are the clump stars. Antony-Twarog et al. (2004) conclude for instance that the high Li stars in NGC 3680 could well be RGB objects rather than clump stars. In view of these considerations we consider the previous hypothesis as rather unlikely.

Another possibility is that some $\mathrm{Li}$ is produced after the end of the first dredge-up. The RGB bump is the most attractive phase where this might take place, as shown by Charbonnel \& Balachandran (2000). At that time, the outward-moving hydrogen burning shell burns through the mean molecular weight discontinuity created by the first dredge-up. An extra-mixing process can then easily connect the ${ }^{3} \mathrm{He}$-rich envelope material to the outer regions of the hydrogen burning shell, enabling Li production to occur. Very high lithium abundances can then be reached, as confirmed by the existence of the socalled "lithium-rich giants". This phase is extremely short-lived and is followed by a phase where deeper mixing destroys the freshly synthesized $\mathrm{Li}$ and lowers the carbon isotopic ratio (e.g., Charbonnel et al. 1998). Palacios et al. (2001) discussed this extra-mixing process in the framework of rotation-induced mixing and called this episode the "lithium flash".

In the present context, the fact that the giant stars in NGC 3680 and IC 4651 have higher Li abundances than their counterparts in NGC 752 (see Pasquini et al. 2001) may be significant. As discussed by Anthony-Twarog \& Twarog (2004), the giants in NGC 3680, and possibly also in IC 4651, could be predominantly first-ascent giants in the bump phase, while the lithium-poor giants in NGC 752 would be clump stars. It is worth noting, however, that these objects are just in the critical mass range where He ignition passes from a degenerate-core flash to a quiescent process. The He flash occurs in less massive stars (found in older clusters like NGC 3680 and IC 4651), which will indeed go through the RGB bump and undergo some Li enrichment. The slightly more massive stars in younger clusters like NGC 752 will ignite He before going through the bump, and thus without undergoing the Li flash. An analysis of the $\mathrm{C}^{12} / \mathrm{C}^{13}$ ratio and $\mathrm{Be}$ abundance in the giants of these three clusters will allow to test if this explanation is correct.

\section{Conclusions}

Our superb high resolution, high $\mathrm{S} / \mathrm{N}$ ratio spectra from the ESO VLT/UVES of 23 stars in the intermediate-age open cluster IC 4651 have enabled us to perform an accurate chemical analysis of stars in critical phases along the CMD of this cluster. Abundances of the Fe-peak and $\alpha$ elements and Li have been derived and interpreted with the help of state-of-the-art evolutionary models incorporating rotational mixing.

We find that the reddening towards the cluster was previously slightly underestimated and we derive a new value of $E(b-y)=0.091$. We confirm that the Fe abundance of IC 4651 is supersolar $([\mathrm{Fe} / \mathrm{H}]=0.10])$, and that the cluster shows a small $\alpha$-element enhancement. For most elements, the abundances of main-sequence and giant stars are in excellent agreement, but $\mathrm{Na}$ is overabundant in the giant stars with respect to the dwarfs. We interpret this as evidence for dredge-up of $\mathrm{Ne}$ processed material.

We discuss Li extensively: the trends of $\mathrm{Li}$ abundance vs. mass and stage of evolution follow very closely the dramatic variations observed in the similar cluster NGC 3680 (Pasquini et al. 2001), confirming that our results for these clusters are typical of disk stars at an age of 1.6-1.8 Gyr. The observed behaviour of $\mathrm{Li}$ in stars more massive than those in the $\mathrm{Li}$ dip is very well reproduced by the most recent stellar evolution models which include rotational mixing. Finally, the pattern of Li depletions in the giant stars is still quite puzzling, and its interpretation critically depends on our ability to discriminate between first-ascent RGB and clump stars.

Further analysis of other elements in IC 4651 is in progress, but the present work has already made IC 4651 a member of the tiny club of open clusters with precise, detailed spectroscopic abundances.

Acknowledgements. We thank S. Meibom for the opportunity to select our programme stars from his thesis in advance of publication, P. Stetson for similarly providing DAOSPEC before publication, and J. Andersen for fruitful discussions and comments on an earlier draft of the paper. B.N. thanks the Carlsberg Foundation, the Danish Natural Science Research Council, the Swedish Research Council, and the Nordic Academy for Advanced Study for financial support. We Finally thank the referee, B. Anthony-Twarog, for useful comments.

\section{References}

Alonso, A., Arribas, R., \& Martinez-Roger, C. 1996, A\&AS, 117, 227 Alonso, A., Arribas, R., \& Martinez-Roger, C. 1999, A\&AS, 140, 261 Anders, E., \& Grevesse, N. 1989, GeoCoA, 53, 197

Anthony-Twarog, B. J., \& Twarog, B. A. 1987, AJ, 94, 1222

Anthony-Twarog, B. J., Mukherjee, K., Twarog, B. A., \& Caldwell, N. 1988, AJ, 95, 1453

Anthony-Twarog, B. J., \& Twarog, B. A. 2000, AJ, 119, 2282

Anthony-Twarog, B. J., \& Twarog, B. A. 2004, AJ, 126, 1000

Arnould, M., Goriely, S., \& Jorissen, A. 1999, A\&A, 347, 572

Ballester, P., Modigliani, A., et al. 2000, The Messenger, 101, 31

Baumüller, D., \& Gehren, T. 1997, A\&A, 325, 1088 
Boesgaard, A. M., \& Tripicco, M. J. 1986, ApJ, 303, 724

Chaboyer, B., Demarque, P., Guenther, D. B., \& Pinsonneault, M. H. 1995, ApJ, 446, 435

Charbonnel, C., \& Balachandran, S. 2000, A\&A, 359, 563

Charbonnel, C., \& Talon, S. 1999, A\&A, 351, 653

Charbonnel, C., \& Vauclair, S. 1992, A\&A, 265, 55

Charbonnel, C., Brown, J.A., Wallerstein, G., 1998, A\&A, 332, 204

Chen, Y. Q., Nissen, P. E., Zhao, G., Zhang, H. W., \& Benoni, T. 2000, A\&AS, 141, 491

Dekker, H., D’ Odorico, S., Kaufer, A., Delabre, B., \& Kotzlowski, H. 2000, SPIE, 4008, 534

Deliyannis, C. P., Pinsonneault, M., \& Charbonnel, C. 2000, The light elements and their evolution, ed. L. da Silva, R. de Medeiros, \& M. Spite, Proc. IAU Symp., 198, 61

do Nascimento, J. D., Charbonnel, C., Lèbre, A., de Laverny, P., \& de Medeiros 2000, A\&A, 357, 931

Edvardsson, B., Andersen, J., Gustafsson, B., et al. 1993, A\&A, 275, 101

Eggen, O. J. 1971, ApJ, 166, 87

El Eid, M. F., \& Champagne, A. E. 1995, ApJ, 451, 298

Gilroy, K. K. 1989, ApJ, 347, 835

Girardi, L., Bressan, A., Bertelli, G., \& Chiosi, C. 2000, A\&AS, 141, 371

Gratton, R. G., Carretta, E., Eriksson, K., \& Gustafsson, B. 1999, A\&A, 350, 955

Gratton, R. 2000, ASP Conf. Ser., 198, 225

Gustafsson, B., Bell, R. A., Eriksson, K., \& Nordlund, A. 1975, A\&A, 42, 407

Kupka, F., Piskunov, N. E., Ryabchikova, T. A., Stempels, H. C., \& Weiss, W. W. 1999, A\&AS, 138, 119

Kurucz, R. L. 1995, ApJ, 452, 102

Lèbre, A., de Laverny, P., de Medeiros, J. R., Charbonnel, C., \& da Silva, L. 1999, A\&A, 345, 936

Lindoff, U. 1972, A\&AS, 7, 231

Maeder, A., \& Meynet, G. 2000, ARA\&A, 38, 143
Meibom, S. 2000, A\&A, 361, 929

Meibom, S., Andersen, J., \& Nordström, B. 2002, A\&A, 386, 187

Nordström, B., Andersen, J., \& Andersen, M. I. 1997, A\&A, 322, 460

Palacios, A., Charbonnel, C., \& Forestini, M. 2001, A\&A, 375, L9

Palacios, A., Talon, S., Charbonnel, C., \& Forestini, M. 2003, A\&A, 399, 603

Pancino, E., Pasquini, L., Hill, V., Ferraro, F., \& Bellazzini, M. 2002, ApJ, 568, L101

Pasquini, L. 2000, The light elements and their evolution, ed. L. da silva, R. de Medeiros, \& M. Spite, Proc. IAU Symp., 198, 269

Pasquini, L., Liu, Q., \& Pallavicini, R. 1994, A\&A, 287, 191

Pasquini, L., Randich, S., \& Pallavicini, R. 2001, A\&A, 374, 1017

Randich, S., Gratton, R. G., Pallavicini, R., \& Pasquini, L. 1999, A\&A, 348, 487

Randich, S., Pasquini, L., \& Pallavicini, R. 2000, A\&A, 356, L25

Randich, S., Primas, F., Pasquini, L., \& Pallavicini, R. 2002, A\&A, 387,222

Sneden, C. A. 1973, ApJ, 184, 839

Soderblom, D. R., Jones, B. F., Balachandran, S., et al. 1993, AJ, 106, 1059

Spite, M. 1967, Ann. Astrophys., 30, 211

Stetson, P., Pancino, E., et al. 2004, in preparation

Talon, S., \& Charbonnel, C. 1998, A\&A, 335, 959

Talon, S., \& Charbonnel, C. 2003, A\&A, 405, 1025

Talon, S., Zahn, J. P., Maeder, G., \& Meynet, A. 1997, A\&A, 322, 209

Takeda, Y., \& Takeda-Hiday, M. 1994, PASJ, 46, 395

Tautvaisiene, G., Edvardsson, B., Tuominen, I., \& Ilyin, I. 2000, A\&A, 360,499

Tosi, M. 2000, in The evolution of the Milky way: stars vs. clusters, ed. F. Matteucci, \& F. Giovannelli (Kluwer), 505

Vauclair, S., Evolution of Stars: The photospheric abundance connection, ed. G. Michaud, \& A. Tutukov, IAU Symp., 145, 327

Wallerstein, G., Böhm-Vitense, E., Vanture, A. D., \& Gonzalez, G. 1994, AJ, 107, 221 